\title{
Association of oxidative stress and memory performance in postmenopausal women receiving estrogen-progestin therapy
}

\author{
Nazlahshaniza Shafin, MD, ${ }^{1}$ Rahimah Zakaria, MBBS, MSc, PhD ${ }^{1}$ \\ Nik Hazlina Nik Hussain, MD, MMed $(O \& G),{ }^{2}$ and Zahiruddin Othman, MD, MMed (Psychiatry) ${ }^{3}$
}

\section{Abstract}

Objective: The aim of this study was to examine the association between changes in blood oxidative stress level/ activity and changes in memory performance among postmenopausal women.

Methods: This study involved 39 postmenopausal women who received estrogen-progestin therapy (EPT) for 16 weeks. Verbal learning and memory performance were assessed using the Malay Version of Auditory Verbal Learning Test before and after 16 weeks of EPT. Oxidative stress levels/activities before and after 16 weeks of EPT were determined using commercially available kits. Data were analyzed using paired $t$ test and $r . P<0.05$ was considered significant.

Results: The activities of glutathione peroxidase and catalase were considerably increased $(P<0.05)$, but the level of 4-hydroxynonenal was notably decreased $(P<0.05)$, after 16 weeks of EPT. There were positive correlations between changes in plasma superoxide dismutase and changes in trial A2 scores $(r=0.36, P<0.05)$, and between changes in the ratio of blood reduced glutathione to oxidized glutathione and changes in trial A2 scores $(r=$ $0.34, P<0.05)$.

Conclusions: Sixteen weeks of EPT increase blood antioxidant capacity. However, most of the changes in oxidative stress level/activity are not significantly associated with changes in the memory performance of postmenopausal women.

Key Words: Estrogen-progestin therapy - Postmenopause - Oxidative stress - Malay Version of Auditory Verbal Learning Test.

$\mathrm{T}$ he effect of hormone therapy (HT) on cognitive function remains inconclusive. Estrogen therapy has been shown to reverse memory impairment associated with estrogen deficiency and to lower the risk of developing dementia in many studies involving postmenopausal women. ${ }^{1-13}$ In contrast, a number of studies found no protective effect of estrogen on either cognitive functioning ${ }^{14-16}$ or dementia. ${ }^{17-19}$ Recently, our group reported improvements in immediate memory, immediate memory after interference, and delayed recall after 16 weeks of estrogen-progestin therapy (EPT) in postmenopausal women. $^{20}$

It has been suggested that cognitive impairment is a consequence of imbalances between local reactive oxygen species and antioxidant capacity within the brain ${ }^{21-23}$ and that an

Received June 20, 2012; revised and accepted October 3, 2012.

From the ${ }^{1}$ Department of Physiology, ${ }^{2}$ Women's Health Development Unit, and ${ }^{3}$ Department of Psychiatry, School of Medical Sciences, Universiti Sains Malaysia, Kubang Kerian, Kelantan, Malaysia.

Funding/support: This work was supported by research university grant 1001/PPSP/811083.

Financial disclosure/conflicts of interest: None reported.

Address correspondence to: Zahiruddin Othman, MD, MMed (Psychiatry), Department of Psychiatry, School of Medical Sciences, Universiti Sains Malaysia, 16150 Kubang Kerian, Kelantan, Malaysia. E-mail: zahir@kb.usm.my unbalanced accumulation of oxidatively modified proteins in the brain potentiates neurodegeneration and impairs cognitive function. $^{21,24}$ The relationship between cognition impairment and oxidative stress is evident from the extensive damage caused by free radicals in age-related neurological conditions such as Alzheimer's disease. ${ }^{25,26}$

Studies have shown that cognitive aging is significantly related to variance in the blood levels of lipid peroxidation. ${ }^{27-31}$ The lipid peroxidation process has few end products, including malondialdehyde, 4-hydroxynonenal (4-HNE), 4-hydroxy2-hexenal, and acrolein. ${ }^{32} 4$-HNE was found to be neurotoxic and to inhibit enzymes critical for neuron survival, including $\mathrm{Na}^{+} / \mathrm{K}^{+}$ATPase, glucose, and glutamate transporters. 4-HNE can lead to changes in the alteration process of neurofibrillary tangles and has been shown to be involved in cognitive impairment. $^{33}$

There are many published studies on the effects of HT on the status of oxidative stress in postmenopausal women, ${ }^{34-40}$ but little is known about its association with cognitive function. Recently, Ansari and Scheff ${ }^{41}$ reported an association between oxidative stress levels and cognitive function in postmortem brain samples. Therefore, the present study aims to examine the association between changes in oxidative stress level/activity and changes in memory performance in postmenopausal women. 


\section{METHODS}

\section{Procedure and design}

The study protocol was approved by the Research and Ethics Committee of Universiti Sains Malaysia. This study involved 39 postmenopausal women aged between 45 and 60 years who had their final menstrual period more than 1 year before study entry. The participants were recruited from the Family Medicine and the Obstetrics and Gynecology Clinic, Hospital Universiti Sains Malaysia. Participants were literate and were not taking any form of medication that could alter their memory function or any other herbal or hormone replacement 3 months before the study. Participants were not chosen if they smoked or had a history of drug or alcohol abuse or a history of serious medical, surgical, mental, or gynecological diseases. Participants underwent physical examinations, including pelvic ultrasonography, to exclude endometrial thickness.

Postmenopausal women who fulfilled the criteria were briefed on the nature of the study, and their consent to participate was taken. During the initial visit, blood was obtained to measure oxidative stress level/activity, and the participants' verbal learning and memory performance were assessed. The participants received Femoston Conti 1/5 (1 mg of 17ß-estradiol plus $5 \mathrm{mg}$ of dydrogesterone) for 16 weeks. Health status, compliance, and any possible adverse effects of EPT were monitored at 8 weeks. After 16 weeks, oxidative stress status and verbal learning and memory performance were reassessed.

\section{Sample collection}

Blood samples were collected into appropriate EDTA bottles to determine oxidative stress level/activity. A total of $150 \mu \mathrm{L}$ of whole blood was used to determine the ratio of reduced glutathione (GSH) to oxidized glutathione (GSSG). The remaining blood was centrifuged at $1,000 \mathrm{~g}$ for 10 minutes at $4^{\circ} \mathrm{C}$ to obtain plasma, aliquoted, and stored at $-80^{\circ} \mathrm{C}$ until assayed.

\section{Determination of memory performance}

Participants' verbal learning and memory performance were assessed using the Malay Version of Auditory Verbal Learning Test (MVAVLT), as previously described. ${ }^{20,42}$ The participants were asked to listen to 15 nouns (list A). List A was read at a rate of one item per second (a tape recorder was used to standardize the delivery rate). The participants had to verbally repeat list $\mathrm{A}$, and this trial was labeled as $\mathrm{A} 1$. The procedures were repeated for another four times. In each trial, the participants were asked to verbally repeat list A after each presentation, and these trials were labeled as A2 to A5. The

TABLE 1. Characteristics of the participants

\begin{tabular}{lr}
\hline Age, mean (SEM), y & $55.31(0.49)$ \\
Menarche age, mean (SEM), y & $13.87(0.27)$ \\
Menopause age, mean (SEM), y & $49.21(0.79)$ \\
Duration of menopause, mean (SEM), y & $6.28(0.73)$ \\
Educational status, n (\%) & \\
$\quad$ Primary & $9(23.1)$ \\
Secondary & $25(64.1)$ \\
Tertiary & $5(12.8)$ \\
\hline
\end{tabular}

TABLE 2. MVAVLT performance of the participants before and after intervention

\begin{tabular}{lcc}
\hline Trial & Before intervention & After intervention \\
\hline A1 & $5.7(0.3)$ & $7.4(0.3)^{a}$ \\
A2 & $8.2(0.4)$ & $9.3(0.3)^{a}$ \\
A3 & $9.5(0.3)$ & $10.2(0.3)$ \\
A4 & $10.1(0.4)$ & $11.3(0.3)^{a}$ \\
A5 & $11.1(0.4)$ & $11.6(0.3)^{a}$ \\
Total learning & $44.4(1.3)$ & $49.7(1.2)^{a}$ \\
B & $5.0(0.3)$ & $5.7(0.3)$ \\
A6 & $9.6(0.4)$ & $10.6(0.3)^{a}$ \\
A7 & $9.9(0.4)$ & $11.4(0.3)^{a}$ \\
Recognition & $13.6(0.3)$ & $14.6(0.1)^{a}$ \\
\hline
\end{tabular}

Values are expressed as mean (SEM).

Preintervention and postintervention scores were compared using paired $t$ test. MVAVLT, Malay Version of Auditory Verbal Learning Test.

${ }^{a} P<0.05$ was considered statistically significant.

second list (or list B) also contains 15 nouns presented to the participants, and the participants were asked to verbally repeat list B (B). A recall of list A was examined without prior presentation of the list by the examiner (A6). After 20 minutes of rest, the recall of list A was repeated also without prior presentation (A7). Lastly, participants had to recognize the words from list A, which were randomly interspersed among other words in a list composed of 30 words.

\section{Determination of blood oxidative stress level/activity}

The blood GSH-to-GSSG ratio and the plasma catalase (CAT), superoxide dismutase (SOD), and glutathione peroxidase (GPx) activities were determined using a commercially available kit (Calbiochem, Darmstadt, Germany). Plasma 4-HNE level was ascertained using the Oxiselect HNE-His adduct ELISA kit (Cell Biolaboratories, San Diego, CA). Procedures for the verification of antioxidant enzymes and lipid oxidative damage were in accordance with the manufacturer's protocol. The intra-assay coefficients of variation for GSH, GSSG, CAT, SOD, GPx, and 4-HNE were $0.96 \%$, $6.45 \%, 5.7 \%, 5.9 \%, 5.6 \%$, and $4.0 \%$, respectively.

\section{Statistical tests}

Results were analyzed using PASW Statistics version 18. Paired $t$ test was used to examine differences in blood oxidative stress values before and after 16 weeks of EPT. $r$ was used to examine the association between changes in blood oxidative stress level/activity and changes in memory performance. $P<0.05$ was considered statistically significant.

\section{RESULTS}

A total of 39 postmenopausal women participated in this study, and the participants' characteristics are summarized in Table 1.

The mean MVAVLT scores before and after 16 weeks of EPT were compared for each trial using paired $t$ test, as shown in Table 2. The mean scores for trials A1, A2, and A4 (immediate memory) were significantly increased when comparing before and after EPT $(P<0.05)$. The mean scores for total learning (summation of scores for trials A1-A5) were 
significantly increased after EPT when compared with the baseline value $(P<0.05)$. The mean scores for trial A6 (immediate memory after interference with list B), trial A7 (delayed memory), and recognition memory were also significantly increased when contrasting before and after EPT $(P<0.05)$. An untreated control group was included for the assessment of memory performance to eliminate the possibility of learning effects. Comparison between the untreated control group and the EPT group showed a significant difference in mean MVAVLT scores for trial A1, trial A5, total learning, trial A6, and trial A7. The complete results had been published earlier. ${ }^{20}$

Blood estradiol and oxidative stress levels/activities measured before and after EPT in postmenopausal women are summarized in Tables 3 and 4, respectively. The serum estradiol levels of postmenopausal women were significantly increased after 16 weeks of EPT $(P<0.05)$.

The plasma CAT and GPx activities were extensively increased after 16 weeks of EPT $(P<0.05)$. The blood GSH-toGSSG ratio, an indicator of oxidative stress, was reduced but was not statistically relevant. There seemed to be almost no change in plasma SOD activity after EPT. Plasma 4-HNE levels, however, showed considerable reduction after 16 weeks of EPT $(P<0.05)$.

$r$ revealed no significant association between the changes in oxidative stress level/activity and the changes in trial scores, except for the changes in trial A2 scores (immediate memory) with changes in plasma SOD $(r=0.36, P<0.05)$ and blood GSH-to-GSSG ratio ( $r=0.34, P<0.05$; Table 5$)$.

\section{DISCUSSION}

The serum estradiol levels of postmenopausal women were notably increased after 16 weeks of EPT. This finding supports the possibility of estrogen contributing to the memory improvement observed in postmenopausal women treated with EPT. ${ }^{20}$ It is believed that estrogen improves cognitive function through its neuroprotective effects, and one of the potential mechanisms involves the powerful antioxidant properties of estrogen. ${ }^{43}$ Estrogen acts directly through its phenolic hydroxyl group at the $\mathrm{C} 3$ position on the A ring of steroid molecules ${ }^{44}$ and indirectly by increasing the levels of endogenous antioxidant enzymes. ${ }^{45} 17 \beta$-Estradiol has a chemical structure that is quite comparable to the lipophilic cellular antioxidant $\alpha$-tocopherol (vitamin E). Both molecules have a phenolic free radical-scavenging group and a lipophilic tail that mediates incorporation into membranes.

TABLE 3. Serum estradiol levels of the participants

\begin{tabular}{|c|c|c|}
\hline & Before intervention & After intervention \\
\hline Estradiol, mean (SEM), pmol/L & $57.37(12.08)$ & $244.03(48.12)^{a}$ \\
\hline
\end{tabular}

TABLE 4. Blood oxidative stress level/activity of the participants before and after intervention

\begin{tabular}{lcc}
\hline & Before intervention & After intervention \\
\hline Blood GSH-to-GSSG ratio & $32.7(8.0)$ & $22.4(6.9)$ \\
Plasma SOD, U/mL & $0.068(0.006)$ & $0.069(0.005)$ \\
Plasma CAT, $\mathrm{nmol} / \mathrm{min} / \mathrm{mL}$ & $4.1(0.4)$ & $6.4(0.5)^{a}$ \\
Plasma GPx, $\mathrm{nmol} / \mathrm{min} / \mathrm{mL}$ & $83.5(5.7)$ & $105.8(8.3)^{a}$ \\
Plasma 4-HNE, $\mu \mathrm{g} / \mathrm{mL}$ & $3.4(0.28)$ & $2.6(0.24)^{a}$ \\
\hline
\end{tabular}

Values are expressed as mean (SEM).

Statistical comparisons before intervention and after intervention were performed using paired $t$ test.

GSH, reduced glutathione; GSSG, oxidized glutathione; SOD, superoxide dismutase; CAT, catalase; GPx, glutathione peroxidase; 4-HNE, 4-hydroxynonenal. ${ }^{a} P<0.05$ was considered statistically significant.

Similar to vitamin E, $17 \beta$-estradiol protects against oxidative damage $^{46}$ through direct quenching of reactive oxygen species, leading to reduced oxidative stress. ${ }^{47}$

A significant increase in CAT activity occurred after 16 weeks of EPT. Increased CAT activity may indicate that this enzyme operated continuously to decompose hydrogen peroxide into harmless products. Recently, Ansari and Scheff ${ }^{41}$ reported that CAT level was significantly decreased in the postmortem brains of participants with mild cognitive impairment and Alzheimer's disease. Thus, it remains possible to suggest that estrogen indirectly increases CAT enzyme activity and improves memory performance in postmenopausal women with EPT. Nevertheless, our findings were contradictory to the findings of previous studies conducted in postmenopausal women. ${ }^{35,38,39}$ Previous studies reported that CAT activity displayed minute differences neither between treated and nontreated postmenopausal women nor between premenopausal and postmenopausal women.

GPx activity was significantly increased after 16 weeks of EPT and was consistent with other studies. ${ }^{36,48}$ GPx level significantly increased in postmenopausal women after 3 months $(\mathrm{n}=22)$ and 6 months $(\mathrm{n}=20)$ of therapy with Premarin $(0.625 \mathrm{mg} / \mathrm{d}$ for $25 \mathrm{~d})$ and Provera $(5 \mathrm{mg} / \mathrm{d}$ for $10 \mathrm{~d}) .{ }^{36}$ In another study, Massafra et $\mathrm{al}^{48}$ reported that erythrocyte GPx was extensively higher in premenopausal women $(\mathrm{n}=90)$ than in postmenopausal women $(\mathrm{n}=60)$. Estrogen may exert antioxidant effects via the modulation of intracellular GPx activity. ${ }^{49}$ However, our results are inconsistent compared with those of previous studies that found no difference in GPx activities in postmenopausal women with and without $\mathrm{HT}^{38,50}$ and in the postmortem brains of participants with mild cognitive impairment and Alzheimer's disease. ${ }^{41}$

The GSH-to-GSSG ratio represents the glutathione redox status, an index of oxidative stress in all types of tissues, cells, and organelles. Under normal physiological conditions, the ratio is above $10 .^{51}$ In this study, the GSH-to-GSSG ratio was above the physiological level both before and after the intervention. However, the ratio was found to be reduced in postmenopausal women after 16 weeks of EPT. The GSHto-GSSG ratio will decrease as a consequence of GSSG 
TABLE 5. $r$ values between changes in blood oxidative stress level/ activity and changes in memory performance

\begin{tabular}{|c|c|c|c|c|c|}
\hline & $\begin{array}{c}\Delta \mathrm{CAT} \\
\text { activity }\end{array}$ & $\begin{array}{c}\Delta \mathrm{GPx} \\
\text { activity }\end{array}$ & $\begin{array}{c}\Delta \text { GSH-to- } \\
\text { GSSG } \\
\text { ratio }\end{array}$ & $\begin{array}{c}\Delta \text { SOD } \\
\text { level }\end{array}$ & $\begin{array}{c}\Delta 4- \\
\text { HNE } \\
\text { level }\end{array}$ \\
\hline$\Delta \mathrm{A} 1$ & -0.05 & -0.08 & 0.05 & 0.14 & 0.05 \\
\hline$\Delta \mathrm{A} 2$ & -0.06 & 0.02 & $0.34^{a}$ & $0.36^{a}$ & -0.03 \\
\hline$\Delta \mathrm{A} 3$ & -0.23 & 0.13 & 0.25 & 0.29 & -0.12 \\
\hline$\Delta \mathrm{A} 4$ & -0.24 & 0.17 & 0.07 & 0.06 & 0.14 \\
\hline$\Delta \mathrm{A} 5$ & 0.25 & -0.15 & -0.20 & -0.22 & 0.12 \\
\hline$\Delta \mathrm{A} 1-\mathrm{A} 5$ & -0.01 & 0.04 & 0.22 & 0.20 & -0.00 \\
\hline$\Delta \mathrm{B} 1$ & -0.29 & -0.22 & 0.19 & 0.22 & -0.45 \\
\hline$\Delta \mathrm{A} 6$ & 0.07 & -0.06 & -0.30 & 0.07 & 0.10 \\
\hline$\Delta \mathrm{A} 7$ & -0.06 & -0.29 & -0.07 & -0.00 & 0.04 \\
\hline$\Delta$ Recognition & 0.16 & 0.01 & 0.21 & 0.07 & -0.26 \\
\hline
\end{tabular}

$\Delta$, change; CAT, catalase; GPx, glutathione peroxidase; GSH, reduced glutathione; GSSG, oxidized glutathione; SOD, superoxide dismutase; 4-HNE, 4-hydroxynonenal.

${ }^{a} P<0.05$ was considered statistically significant.

accumulation $^{51,52}$ when exposed to increased oxidative stress. Through EPT, depletion of GSH, together with evidence of increased GPx, suggests an ongoing process to detoxify oxidative stress. The present study revealed a significant positive correlation between the changes in the blood GSH-to-GSSG ratio and the changes in trial A2 scores. This finding implies that when the oxidative stress level decreased (as shown by an increased blood GSH-to-GSSG ratio), immediate memory performance improved (as shown by increased trial A2 scores). This finding is supported by previous studies, which revealed that older persons in urban areas had more oxidative stress and a higher risk of developing cognitive impairment when compared with older persons living in rural communities. ${ }^{53}$

The plasma SOD level was almost unchanged after 16 weeks of EPT, but there was a significant positive correlation between the changes in plasma SOD level and the changes in trial A2 scores. A study conducted by Ansari and Scheff ${ }^{41}$ revealed that the changes in antioxidant levels (ie, GSH, SOD, and CAT) strongly correlated with the Mini Mental State Examination score, supporting the idea that major changes in oxidative stress compose an early event that plays an imperative role in the progression of the disease. Our results are inconsistent, however, with those of previous studies, which showed either an increase or a decrease in SOD levels with HT. Unfer et $\mathrm{al}^{39}$ showed an increase in SOD levels to premenopausal levels in postmenopausal women treated with HT compared with postmenopausal women without HT. Other studies, however, showed a reduction in SOD levels after 6 months of HT. ${ }^{36}$

The present study revealed a reduction in plasma levels of 4-HNE, a marker for lipid peroxidation, after 16 weeks of EPT, consistent with the findings from a previous study. ${ }^{41}$ 4-HNE has been shown to be associated with cognitive impairment. ${ }^{33}$ It is consistent with our findings that a decrease in plasma 4-HNE levels was related to improved memory performance among postmenopausal women treated with EPT.

In the present study, the increase in the main antioxidant enzyme activities reduces the production of hydrogen peroxide and reactive hydroxyl radicals and thus reduces oxida- tive stress damage. The changes in peripheral antioxidant activities may reflect similar changes in the brain regions related to the memory function of postmenopausal women treated with EPT. These findings may shed light on the possible neuroprotective roles of estrogen in memory function, consistent with previous imaging studies. Positron emission tomography studies indicate that HT modulates the pattern of regional cerebral blood flow activation in brain regions that play critical roles in memory processing and shows that hypoperfusion occurs in individuals who are at an increased risk of developing Alzheimer's disease ${ }^{54-56}$ and who are in the early stages of the disease. ${ }^{57}$ Shaywitz et $\mathrm{al}^{58}$ reported HT effects on functional magnetic resonance imaging activation patterns during the performance of verbal and visual working memory tasks, similar to the findings in positron emission tomography studies.

Limitations of the present study include the absence of an age-matched control group for changes in blood oxidative stress level/activity and the fact that the effects on blood oxidative stress level/activity were not specified to different stages of menopause. We were unable to control for certain factors, such as diet, exercise, and health status, that were known to alter oxidative stress level/activity and in turn may exert direct or indirect effects on memory performance. Therefore, in the present study, the baseline (before intervention) values of the oxidative stress markers were used as control values for each participant.

We suggest future causal relationship studies using a larger sample size with age-matched controls and a longer duration of EPT to confirm the association between the changes in oxidative stress and the changes in the memory performance of postmenopausal women at different stages of menopause.

\section{CONCLUSIONS}

Sixteen weeks of EPT reduce oxidative stress and improve memory performance in postmenopausal women. It is unclear whether the direct antioxidant properties of estrogen or its indirect effects through increased plasma CAT and GPx activities are capable of reducing oxidative stress. The association between the changes in the plasma level/activity of oxidative stress and the changes in memory performance is unremarkable. Only plasma SOD levels and the blood GSHto-GSSG ratio are positively correlated with the changes in immediate memory performance.

\section{REFERENCES}

1. Phillips SM, Sherwin BB. Effects of estrogen on memory function in surgically menopausal women. Psychoneuroendocrinology 1992; 17:485-495.

2. Phillips SM, Sherwin BB. Variations in memory function and sex steroid hormones across the menstrual cycle. Psychoneuroendocrinology 1992; 17:497-506.

3. Birge SJ. Is there a role for estrogen replacement therapy in the prevention and treatment of dementia? J Am Geriatr Soc 1996;44:865-870. 
4. Paganini-Hill A, Henderson VW. Estrogen replacement therapy and risk of Alzheimer disease. Arch Intern Med 1996;156:2213-2217.

5. Sherwin BB, Tulandi T. "Add-back" estrogen reverses cognitive deficits induced by a gonadotropin-releasing hormone agonist in women with leiomyomata uteri. J Clin Endocrinol Metab 1996;81:2545-2549.

6. Tang MX, Jacobs D, Stern Y, et al. Effect of oestrogen during menopause on risk and age at onset of Alzheimer's disease. Lancet 1996;348: 429-432.

7. Kawas C, Resnick S, Morrison A, et al. A prospective study of estrogen replacement therapy and the risk of developing Alzheimer's disease: The Baltimore Longitudinal Study of Aging. Neurology 1997;48:1517-1521.

8. Baldereschi M, Di Carlo A, Lepore V, et al. Estrogen-replacement therapy and Alzheimer's disease in the Italian Longitudinal Study on Aging. Neurology 1998;50:996-1002.

9. Jacobs DM, Tang MX, Stern Y, et al. Cognitive function in nondemented older women who took estrogen after menopause. Neurology 1998;50: 368-373.

10. Sherwin BB. Estrogen and/or androgen replacement therapy and cognitive functioning in surgically menopausal women. Psychoneuroendocrinology 1998:13:345-357.

11. Resnick SM, Maki PM, Golski S, Kraut MA, Zonderman AB. Effects of estrogen replacement therapy on PET cerebral blood flow and neuropsychological performance. Horm Behav 1998;34:171-182.

12. Maki PM, Resnick SM. Longitudinal effects of estrogen replacement therapy on PET cerebral blood flow and cognition. Neurobiol Aging 2000;21:373-383.

13. Kampen DL, Sherwin BB. Estrogen use and verbal memory in healthy postmenopausal women. Obstet Gynecol 1994;83:979-983.

14. Cauley JA, Cummings SR, Black DM, Mascioli SR, Seeley DG. Prevalence and determinants of estrogen replacement therapy in elderly women. Am J Obstet Gynecol 1990;163:1438-1444.

15. Barrett-Connor E, Kritz-Silverstein D. Estrogen replacement therapy and cognitive function in older women. JAMA 1993;269:2637-2641.

16. Matthews K, Cauley J, Yaffe K, Zmuda JM. Estrogen replacement therapy and cognitive decline in older community women. $J$ Am Geriatr Soc 1999;47:518-523.

17. Brenner DE, Kukull WA, Stergachis A, et al. Postmenopausal estrogen replacement therapy and the risk of Alzheimer's disease: a populationbased case-control study. Am J Epidemiol 1994;140:262-267.

18. Henderson VW, Paganini-Hill A, Miller BL, et al. Estrogen for Alzheimer's disease in women: randomized, double-blind, placebocontrolled trial. Neurology 2000;54:295-301.

19. Mulnard RA, Cotman CW, Kawas C, et al. Estrogen replacement therapy for treatment of mild to moderate Alzheimer disease: a randomized controlled trial. Alzheimer's Disease Cooperative Study. JAMA 2000; 283:1007-1015.

20. Othman Z, Shafin N, Zakaria R, Hussain NHN, Mohammad WMZW. Improvement in immediate memory after 16 weeks of tualang honey (Agro Mas) supplement in healthy postmenopausal women. Menopause 2011;8:1219-1224

21. Davies KJ. Oxidative stress, antioxidant defenses, and damage removal, repair, and replacement systems. IUBMB Life 2000;50:279-289.

22. Ames BN. Low micronutrient intake may accelerate the degenerative diseases of aging through allocation of scarce micronutrients by triage. Proc Natl Acad Sci U S A 2006;103:17589-17594.

23. Corbetta M, Patel G, Shulman GL. The reorienting system of the human brain: from environment to theory of mind. Neuron 2008;58:306-324

24. Radak Z, Kumagai S, Nakamoto H, Goto S. 8-Oxoguanosine and uracil repair of nuclear and mitochondrial DNA in red and white skeletal muscle of exercise-trained old rats. $J$ Appl Physiol 2007;102:1696-1701.

25. Coyle JT, Puttfarcken P. Oxidative stress, glutamate, and neurodegenerative disorders. Science 1993;262:689-695.

26. Smith MA, Perry G, Richey PL, et al. Oxidative damage in Alzheimer's. Nature 1996;382:120-121.

27. Berr C, Richard MJ, Roussel AM, Bonithon-Kopp C. Systemic oxidative stress and cognitive performance in the population-based EVA study. Free Radic Biol Med 1998;24:1202-1208.

28. Waddington E, Croft KD, Clarnettte R, Mori T, Martins R. Plasma F2isoprostanes levels are increased in Alzheimer's disease: evidence of increased oxidative stress in vivo. Alzheimer's Rep 1999;2:277-282.

29. Tuppo EE, Forman LJ, Spur BW, Chan-Ting RE, Chopra A, Cavalieri TA. Sign of lipid peroxidation as measured in the urine of patients with probable Alzheimer's disease. Brain Res Bull 2001;54:565-568.
30. Montine TJ, Neely MD, Quinn JF, et al. Lipid peroxidation in aging brain and Alzheimer's disease. Free Radic Biol Med 2002;33:620-626.

31. Pratico D, Clark CM, Liun F, Lee VYM, Trojanowski JQ. Increase of brain oxidative stress in mild cognitive impairment. Arch Neurol 2002; 59:972-976.

32. Esterbauer H, Schaur RJ, Zollner H. Chemistry and biochemistry of 4 hydroxynonenal, malonaldehyde and related aldehydes. Free Radic Biol Med 1991;11:81-128.

33. Williams TI, Lynn BC, Markesbery WR, et al. Increased levels of 4hydroxynonenal and acrolein, neurotoxic markers of lipid peroxidation, in the brain in mild cognitive impairment and early Alzheimer's disease. Neurobiol Aging 2006;27:1094-1099.

34. Darabi M, Anti M, Movahedian A, Zarean E, Panjehpour M, Rabbani M. Effect of hormone replacement therapy on total serum anti-oxidant potential and oxidized LDL/32-glycoprotein I complexes in postmenopausal women. Endocr J 2010;57:1029-1034.

35. Gürdöl F, Oner-Yyidothan Y, Yalcýn O, Genc S, Buyru F. Changes in enzymatic antioxidant defense system in blood and endometrial tissues of women after menopause. Res Commun Mol Pathol Pharmacol 1997; 97:38-46.

36. Akçay T, Dinçer Y, Kayali R, Colgar U, Oral E, Cakatay U. Effects of hormone replacement therapy on lipid peroxides and oxidation system in postmenopausal women. J Toxicol Environ Health A 2000;59:1-5.

37. Ke RW, Todd Pace D, Ahokas RA. Effect of short-term hormone therapy on oxidative stress and endothelial function in African American and Caucasian postmenopausal women. Fertil Steril 2003;79:1118-1122.

38. Naziroglu M, Simsek M, Simsek H, Aydilek N, Özcan Z, Atilgan R. The effects of hormone replacement therapy combined with vitamins $\mathrm{C}$ and $\mathrm{E}$ on antioxidants levels and lipid profiles in postmenopausal women with type 2 diabetes. Clin Chim Acta 2004;344:63-71.

39. Unfer TC, Conterato GMM, Da Silva JCN, Duarte MMMF, Emanuelli T. Influence of hormone replacement therapy on blood antioxidant enzymes in menopausal women. Clin Chim Acta 2006;369:73-77.

40. Inal M, Sunal E, Kanbak G, Zeytinoğlu S. Effects of postmenopausal hormone replacement and $[\alpha]$-tocopherol on the lipid profiles and antioxidant status. Clin Chim Acta 1997;268:21-29.

41. Ansari MA, Scheff SW. Oxidative stress in the progression of Alzheimer disease in the frontal cortex. J Neuropathol Exp Neurol 2010;69:155-167.

42. Ruzita J, Zahiruddin O, Kamarul IM, Muhammad Najib MA. Validation of the Malay Version of Auditory Verbal Learning Test (MVAVLT) among schizophrenia patients in Hospital Universiti Sains Malaysia (HUSM), Malaysia. Online ASEAN J Psychiatry 2009;10:54-74.

43. Genazzani AR, Pluchino N, Luisi S, Luisi M. Estrogen, cognition and female ageing. Hum Reprod Update 2007;13:175-187.

44. Moosmann B, Behl C. The antioxidant neuroprotective effects of estrogens and phenolic compounds are independent from their estrogenic properties. Proc Natl Acad Sci U S A 1999;96:8867-8872.

45. Baeza I, Fdez-Tresguerres J, Ariznavarreta C, De la Fuente M. Effects of growth hormone, melatonin, oestrogens and phytoestrogens on the oxidized glutathione (GSSG)/reduced glutathione (GSH) ratio and lipid peroxidation in aged ovariectomized rats. Biogerontology 2010;11: 687-701.

46. Behl C, Skutella T, Lezoualc'h F, et al. Neuroprotection against oxidative stress by estrogens: structure-activity relationship. Mol Pharmacol 1997; 51:535-541.

47. Kumar S, Lata K, Mukhopadhyay S, Mukherjee TK. Role of estrogen receptors in pro-oxidative and anti-oxidative actions of estrogens: a perspective. Biochim Biophys Acta 2010;1800:1127-1135.

48. Massafra C, Gioia D, De Felice C, Muscettola M, Longini M, Buonocore G. Gender-related differences in erythrocyte glutathione peroxidase activity in healthy subjects. Clin Endocrinol 2002;57:663-667.

49. Massafra C, Buonocore G, Gioia D, Sargentini I, Farina G. Effects of estradiol and medroxyprogesterone-acetate treatment on erythrocyte antioxidant enzyme activities and malondialdehyde plasma levels in amenorrhoic women. J Clin Endocrinol Metab 1997;82:173-175.

50. Özden S, Dildar K, Kadir YH, Gülizar K. The effects of hormone replacement therapy on lipid peroxidation and antioxidant status. Maturitas 2001;38:165-170.

51. Wu G, Fang YZ, Yang S, Lupton JR, Turner ND. Glutathione metabolism and its implications for health. J Nutr 2004;134:489-492.

52. Bains JS, Shaw CA. Neurodegenerative disorders in humans: the role of glutathione in oxidative stress-mediated neuronal death. Brain Res Rev 1997;25:335-358 
53. Sánchez-Rodríguez MA, Santiago E, Arronte-Rosales A, VargasGuadarrama LA, Mendoza-Núnez VM. Relationship between oxidative stress and cognitive impairment in the elderly of rural vs. urban communities. Life Sci 2006;78:1682-1687.

54. Ansari MA, Roberts KN, Scheff SW. Oxidative stress and modification of synaptic proteins in hippocampus after traumatic brain injury. Free Radic Biol Med 2008;45:443-452.

55. Small GW, Mazziotta JC, Collins MT, et al. Apolipoprotein E type 4 allele and cerebral glucose metabolism in relatives at risk for familial Alzheimer disease. JAMA 1995;273:942-947.
56. Reiman EM, Caselli RJ, Yun LS, et al. Preclinical evidence of Alzheimer's disease in persons homozygous for the epsilon 4 allele for apolipoprotein E [see comments]. N Engl J Med 1996; 334:752-758.

57. Jagust WJ, Eberling JL, Richardson BC, et al. The cortical topography of temporal lobe hypometabolism in early Alzheimer's disease. Brain Res 1993;629:189-198.

58. Shaywitz SE, Shaywitz BA, Pugh KR, et al. Effect of estrogen on brain activation patterns in postmenopausal women during working memory tasks. JAMA 1999;281:1197-1202. 